\title{
Influence du parcage nocturne et du fractionnement de la surface à pâturer sur l'ingestion chez les génisses créoles conduites à l'attache
}

\author{
M Boval ${ }^{1}$, JL Peyraud ${ }^{2}$, A Xandé 1 \\ 1 Station de zootechnie, Inra, BP 1232, 97185 Pointe-à-Pitre cedex; \\ 2 Station de recherches sur la vache laitière, Inra, 35590 Saint-Gilles, France
}

(Reçu le 20 juillet 1994 ; accepté le 21 août 1995)

\begin{abstract}
Résumé - L'objectif de cette étude était de tester les effets du parcage nocturne et du fractionnement de la surface à pâturer sur l'ingestion chez les génisses créoles conduites à l'attache sur des savanes à Dichanthium sp. Six génisses ont été maintenues au pâturage i) pendant 24 heures, ii) pendant 11 heures de la période diurne, iii) pendant ces mêmes 11 heures mais avec la surface fractionnée en deux parties, la première disponible le matin de $7 \mathrm{~h} 00$ à $12 \mathrm{~h} 30$ et la seconde l'après-midi de $12 \mathrm{~h} 30$ à $18 \mathrm{~h} 00$. Les génisses ne pâturant que pendant la journée ont été attachées pour la nuit sur une aire en terre battue sans accès à du fourrage. L'essai a été réalisé selon un schéma en carré latin répété deux fois (six périodes) avec trois lots de deux génisses. Les surfaces et les quantités d'herbe offertes étaient identiques pour les trois traitements. Les quantités ingérées ont été calculées à partir de la quantité de matière organique excrétée et de la digestibilité de la matière organique du Dichanthium $\mathrm{sp}$, estimée à partir de la teneur en matières azotées des fèces. La présence sur le pâturage pendant tout le nycthémère a permis d'accroître les quantités ingérées comparé au parcage nocture $(4,3$ versus 3,6 $\mathrm{kg} \mathrm{MO} / \mathrm{jour}$ respectivement). Cet accroissement de l'ingestion s'explique par l'activité de pâturage nocturne car la durée de pâturage ( 330 minutes) et la fréquence des bouchées (53/minute) n'ont pas varié durant la phase diurne entre les trois traitements. La durée de pâturage nocturne a été de 100 minutes. Le fractionnement de la surface offerte n'a pas entraîné de modifications de l'ingestion ni du comportement alimentaire au cours de la période diurne.
\end{abstract}

ingestion / pâturage / parcage nocturne / fractionnement / attache / Dichanthium sp

Summary - Effect of nocturnal enclosing and splitting offered area on herbage intake of tethered creole heifers. The experiment was aimed at studying the effect of nocturnal enclosing and splitting the offered area during the day upon intake and feeding behaviour of creole heifers grazing Dichanthium $s p$ pastures. The three treatments were i) a whole area available during $24 \mathrm{~h}$ (S24); ii) a same whole area available during $11 \mathrm{~h}$ (S11); iii) a same area available during $11 \mathrm{~h}$, but divided into two parts (F11), the first available from 700 to 1200 hours, the second one from 1230 to 1800 hours. Offered area and daily herbage allowance were similar for the three treatments. All the heifers were 
moved to a fresh area every day at 700 hours. The treatments $S 11$ and F11 were tethered outside pasture at night from 1800 hours and no supplementary feed was available. Three groups of two heifers (mean weight $162 \mathrm{~kg}$ ) were used in a $3 \times 3$ latin square design repeated two times (six periods). Organic matter intake $(\mathrm{MOI}, \mathrm{kg}$ ) was calculated from faecal organic matter output and organic matter digestibility ( $(\mathrm{MO})$ ). dMO was predicted from faecal crude protein content (MATf, \% MO) in feeding trials with similar swards: $d M O=0.983-4.002 / M A T f ; R S D=0.023 ; \mathrm{R}^{2}=0.84$. Total faecal excretion was collected for 4 days. The mean herbage allowance was $11.6 \mathrm{~kg}$ OM/heifer/days. MOI was greater in treatment S24 (4.3 $\mathrm{kg} O M, P<0,001)$ than in treatment S11 and $F 11(3.7 \mathrm{~kg}$ OM on the mean). This was related to a higher grazing time in $\mathrm{S} 24$. Indeed, daylight grazing times (mean $320 \mathrm{mn}$ ) and biting rates (53 bites/min) were not affected by treatment, nocturnal grazing time was $100 \mathrm{~min}$ for treatment $S 24$. However, biting rates during the night (42 bites $/ \mathrm{min}, \mathrm{P}<0.001$ ) was lower than during the daylight period. Splitting the daylight offered area did not affect herbage intake or feeding behaviour.

intake / pasture / nocturnal enclosing splitting up / tethering / Dichanthium sp

\section{INTRODUCTION}

En Guadeloupe, le pâturage à l'attache des bovins créoles sur des savanes naturelles à Dichanthium sp est le mode de conduite le plus répandu. Ce mode traditionnel d'utilisation de la savane permet une gestion intensive du milieu et rend possible l'utilisation de surfaces exiguës et incultes (Buisson et Salas, 1985 ; Salas et Sheikboudou, 1988). Une enquête a mis en évidence la diversité des pratiques de ce mode de conduite en Guadeloupe (Boval et al, 1995a). En particulier, il est apparu que les animaux sont attachés avec des chaînes de longueur variable, ce qui correspond le plus souvent à des variations de quantités d'herbe offerte. La fréquence de déplacement sur de nouvelles surfaces est également très variable. Les animaux sont déplacés de 1 à 4 fois par jour, l'abreuvement ayant lieu au cours de ces déplacements. Enfin les animaux sont parfois parqués pour la nuit avec des chaînes très courtes, hors de la savane pour la récupération de fumier. C'est la pratique du «piquet dortoir».

Les effets de ces diverses modalités de conduite sur l'ingestion au pâturage n'ont jamais été quantifiés. La pratique du piquet dortoir pourrait stimuler l'appétit des animaux selon les éleveurs enquêtés (Boval et al, 1995a). À l'inverse, elle pourrait aussi limiter les quantités ingérées en restreignant notamment la durée de pâturage à la phase diurne. Par ailleurs le déplacement des animaux plusieurs fois par jour sur différents pâturages et le fractionnement de la surface quotidienne à pâturer qui en résulte pourrait accroître l'ingestion ainsi que cela est observé à l'auge (Chenost et Demarquilly, 1982 ; Ikhatua et al, 1987).

L'objectif de cet essai est de tester les effets de la pratique du piquet dortoir et du fractionnement de la surface à pâturer pendant la journée sur l'ingestion et le comportement alimentaire de génisses créoles conduites à l'attache sur des savanes naturelles à Dichanthium $\mathrm{sp}$.

\section{MATÉRIEL ET MÉTHODES}

\section{Traitements et schéma expérimental}

Les trois traitements correspondaient à une même surface à pâturer qui était offerte selon trois modalités : i) les génisses sont présentes sur la surface pendant 24 heures (traitement S24) et sont déplacées sur une nouvelle surface tous les matins à $7 \mathrm{~h} 00$; ii) la surface n'est disponible que pendant les 11 heures de la période diurne (traitement S11) soit de $7 \mathrm{~h} 00$ à $18 \mathrm{~h} 00$; iii) la surface est disponible pendant les mêmes heures mais est fractionnée en deux surfaces égales (traitement F11), les génisses étant présentes 
sur un premier cercle de pâturage de $7 \mathrm{~h} 00$ à $12 \mathrm{~h} 30$ et sur un second de $12 \mathrm{~h} 30$ à $18 \mathrm{~h} 00$. Dans les traitements $S 11$ et $F 11$, les génisses sont mises au piquet dortoir et n'ont alors accès à aucun fourrage.

L'essai a été conduit selon un schéma en carré latin $3 \times 3$ répété deux fois (six périodes de mesure). Chaque période expérimentale a duré 11 jours, les mesures étant réalisées durant les quatre derniers jours après sept jours d'adaptation des génisses aux traitements expérimentaux. Trois lots de deux génisses créoles ont été utilisés, les génisses étaient âgées de 10 mois et pesaient $160 \mathrm{~kg}$ en moyenne au début de l'essai. Les quantités d'herbe offertes étaient identiques pour tous les traitements.

\section{Conduite des parcelles expérimentales et du pâturage}

L'essai s'est déroulé d'avril à juillet 1993 sur une savane à Dichanthium sp au domaine Inra de Gardel, situé à une altitude de $40 \mathrm{~m}$, sur des sols de type vertique à montmorillonite. La savane a été divisée en deux lots de 11 sous-parcelles de $600 \mathrm{~m}^{2}$ chacune, cette surface devant permettre une journée de pâturage pour les six génisses. Chaque lot de parcelles a été utilisé alternativement au cours des périodes de mesure. Afin d'exploiter l'herbe à un âge constant durant toute la période de mesure, chaque sous-parcelle a été fauchée 14 jours avant et 14 jours après l'exploitation par les génisses. La fauche a été réalisée à une hauteur de 3 à $5 \mathrm{~cm}$ du sol, avec une tondeuse automotrice munie d'un bac de récolte de l'herbe.

Toutes les sous-parcelles ont été fertilisées 1 mois avant le début de l'essai avec $30 \mathrm{~kg}$ d'azote/ha. Chacune a ensuite été fertilisée au moment des fauches avec $20 \mathrm{~kg}$ d'azote/ha. La fertilisation a été interrompue à la quatrième période de mesure, en raison d'une production très importante de biomasse. L'irrigation des parcelles a été effectuée afin de pallier les variations de la pluviométrie entre les périodes de mesure. Elle a été réalisée à 2 jours de repousse, puis 2 jours après le passage des génisses. Les durées d'irrigation ont été calculées en fonction d'un bilan hydrique réalisé tous les 15 jours à partir de l'évapo-transpiration et de la pluviométrie mesurées sur les parcelles expérimentales.
A chacune des périodes, les surfaces offertes ont été déterminées en fonction de la quantité d'herbe présente afin d'offrir $12 \mathrm{~kg}$

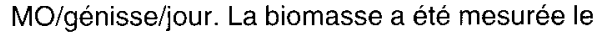
premier jour de la période puis à j8, j9 et j10. Les surfaces ont été ajustées par la longueur de la chaîne et en prenant en compte la longueur du cou de l'animal, estimée à $70 \mathrm{~cm}$. Les longueurs de chaîne ont été identiques entre les traitements $\mathrm{S} 24$ et S11. Elles ont été réduites dans le traitement F11 afin d'offrir deux disques de pâturage ayant chacun une surface deux fois plus faible que pour S24 et S11. Pour les traitements S11 et $\mathrm{F} 11$ les génisses étaient mises de $18 \mathrm{~h} 00$ à $7 \mathrm{~h} 00$ au piquet dortoir sur une aire en terre battue. L'abreuvement a été réalisé entre $12 \mathrm{~h} 00$ et $13 \mathrm{~h} 00$ sur les parcelles, individuellement et à volonté. Les génisses ont été pesées à la fin de chaque période de mesure, le matin à $7 \mathrm{~h} 00$. Elles ont été traitées contre les tiques par aspersion (au deltaméthrine) au moment des pesées. Un traitement anthelmintique (l'ivermectine) a été effectué tous les mois.

\section{Estimation des quantités ingérées}

Les quantités de matière organique ingérées (MOl) par génisse et par jour ont été déterminées à partir des quantités de matière organique fécale excrétées (MOF) et de la digestibilité de la matière organique ( $\mathrm{dMO}$ ) du fourrage ingéré : $\mathrm{MOI}=\mathrm{MOF} /(1-\mathrm{dMO})$.

Les quantités de MOF ont été mesurées par récolte totale et manuelle des fèces sur le sol. Les fèces ont été ramassées trois fois par jour à $7 \mathrm{~h} 00,12 \mathrm{~h} 00$ et $17 \mathrm{~h} 00$ afin de limiter leur piétinement. La récolte manuelle a été facilitée par la teneur élevée en matière sèche des bouses (en moyenne $18,4 \%$ ). La fraction de fèces qui n'a pu être récoltée sur les parcelles a été estimée à environ $2 \%$ de la MS (Boval, non publié). La teneur en MS a été déterminée chaque jour à partir de deux échantillons de $500 \mathrm{~g}$. Pour les analyses chimiques, les échantillons journaliers ont été regroupés par génisse en pondérant pour les quantités journalières de matière sèche excrétées.

La digestibilité de la matière organique (dMO) a été estimée à partir de la teneur en matières azotées des fèces (MATf $f$ de l'échantillon moyen individuel d'après l'équation établie par Boval et al (1996) sur Dichantium sp : 
$\mathrm{dMO}=0,983-4,002 / \mathrm{MAT} f$

$\left(\mathrm{ETR}=0,0230 ; R^{2}=0,84 ; n=38\right)$

La pertinence de l'estimation de $\mathrm{MOI}$ a été validée en comparant les apports énergétiques estimés et les besoins théoriques des génisses. Les apports en énergie ont été calculés en multipliant les quantités de MOI par la valeur énergétique du Dichanthium sp. Celle-ci a été estimée à partir d'une relation entre la dMO et la valeur énergétique (UFL/kg MO) de quatre fourrages tropicaux (Dichanthium sp, Digitaria decumbens, Digitaria swazilendensis, Panicum maximum) à différents âges de repousse (Aumont et al, 1991):

$\mathrm{UFL}=1,55 \times \mathrm{dMO}-0,255$

$\left(R^{2}=0,98 ; \mathrm{ETR}=0,009\right)$

Les besoins en énergie nette des génisses (UFL $/ k g \mathrm{P}^{0,75}$ ) au pâturage ont été estimés selon l'équation de Troccon (1987) à partir du gain de poids moyen quotidien (GMQ en kilo). Ces besoins ont été majorés de $20 \%$ pour les dépenses liées à la thermorégulation au pâturage (Berbigier, 1988) :

$\mathrm{UFL}\left(/ \mathrm{kg} \mathrm{P} \mathrm{P}^{0,75}\right)=\left(0,044+0,0415 \times \mathrm{GMQ}^{1,4}\right) \times 1,2$

\section{Enregistrement du comportement alimentaire}

Le comportement alimentaire a été enregistré par observation visuelle des génisses par deux observateurs situés à une distance de 3 à $6 \mathrm{~m}$ des génisses. Celles-ci avaient été habituées au préalable à la présence des observateurs.

Les observations ont été réalisées de $7 \mathrm{~h} 00$ à $12 \mathrm{~h} 00$ et de $13 \mathrm{~h} 00$ à $18 \mathrm{~h} 00$, pendant 2 jours consécutifs à chaque période. Les activités de pâturage, de rumination et de repos ont été notées toutes les 10 minutes pour chaque génisse. Le pâturage a été défini comme l'arrachage et la recherche de talles, l'animal ayant la tête au niveau de la prairie (Jamieson et Hodgson, 1979). La fréquence de bouchées (nombre/ minute) a été estimée toutes les 10 minutes par comptage du nombre de bouchées réalisé en 1 minute, pour les génisses qui pâturaient. La bouchée a été définie comme l'arrachage du fourrage et comptabilisée à partir du bruit de l'arrachage de l'herbe.
Le comportement alimentaire des génisses du traitement S24 a été observé durant une nuit (de $18 \mathrm{~h} 00$ à $7 \mathrm{~h} 00$ ) lors des deux dernières périodes de mesure (périodes 5 et 6 ) et selon les mêmes modalités que les observations diurnes. L'observation des activités de pâturage, de rumination et de repos ont nécessité un éclairage furtif des génisses toutes les 10 minutes. Cet éclairage ne semble pas avoir perturbé les animaux. La fréquence des bouchées a pu être comptabilisée sans éclairage.

La durée de chaque activité a été estimée en multipliant par 10 minutes le nombre d'occurrences. Ces durées ont été calculées pour l'ensemble de la phase diurne et pendant la phase nocturne durant les périodes 5 et 6 . Elles ont aussi été calculées pour chaque tranche horaire. Le nombre de bouchées durant la période diurne (exceptée durant la tranche horaire $12 \mathrm{~h} 00$ à $13 \mathrm{~h} 00$ ) et la période nocturne a été déterminé en multipliant la fréquence moyenne des bouchées par la durée de pâturage de chacune de ces deux phases. Pour le traitement S24, le nombre total de bouchées a été calculé pour les périodes 5 et 6 en sommant le nombre de bouchées diurnes et nocturnes. Le poids moyen de chaque bouchée a été estimé en divisant les quantités ingérées par le nombre total de bouchées.

\section{Biomasse offerte et caractérisation du couvert végétal}

La biomasse présente sur les cercles de pâturage offerts aux génisses a été mesurée au cours des jours 8,9 et 10 de chacune des périodes. Pour chaque génisse, dix bandes de $0,03 \mathrm{~m}^{2}$ $(30 \times 10 \mathrm{~cm})$ réparties de manière homogène sur la surface offerte ont été coupées au niveau du sol à l'aide d'une minitondeuse. Dans le cas du traitement $F 11$, cinq bandes ont été fauchées sur chacune des deux surfaces occupées par jour. Les bandes ont été pesées individuellement puis regroupées par traitement et par jour. Deux échantillons de $300 \mathrm{~g}$ ont été constitués pour la détermination de la MS et de la composition chimique. La composition morphologique de la biomasse proposée aux génisses a été déterminée à partir d'un troisième échantillon de $300 \mathrm{~g}$, en séparant manuellement les différentes parties de la plante entière (tiges, feuilles, débris) qui ont été séchées puis pesées. 


\section{Analyses chimiques}

Les échantillons de fourrage et de fèces ont été séchés à $80^{\circ} \mathrm{C}$ pendant 48 heures et broyés à travers une grille à mailles de $0,75 \mathrm{~mm}$. Les échantillons ont été analysés pour déterminer les teneurs en cendres (MM), en matières azotées totales (MAT), en parois totales (NDF), et en lignocellulose (ADF). La digestibilité in vitro de la matière sèche (DIV) a été déterminée pour les échantillons de fourrage.

La teneur en cendres a été déterminée par calcination des échantillons à $550^{\circ} \mathrm{C}$ pendant 8 heures. Les teneurs en MAT ont été déterminées par la méthode Kjeldahl. Après minéralisation de l'azote, la distillation de l'ammoniaque a été réalisée à l'aide d'un générateur de vapeur (Vapodest) couplé à un titrimètre automatique. Le NDF et l'ADF ont été déterminés selon la méthode de Van Soest (1963) automatisée à l'aide du fiberteck M6 (Técator). La DIV a été estimée par la méthode de Tilley et Terry (1963).

\section{Analyses statistiques}

Les données ont été analysées selon un schéma en carré latin avec deux répétitions avec la procédure GLM de SAS (1988). Les variables liées à l'estimation des quantités ingérées et à l'analyse du comportement alimentaire ont été analysées selon le modèle:

$$
\mathrm{Y}_{i j k l}=\mathrm{m}+\mathrm{A}_{i}+\mathrm{B}\left(\mathrm{A}_{i}\right)_{j}+\mathrm{C}_{k}+\mathrm{D}_{1}+\mathrm{e}_{i j k l}
$$

où $Y$ est le paramètre étudié ; $m$, la valeur moyenne du paramètre ; $A_{i}$ l'effet du carré latin $(i=2) ; \mathrm{B}\left(\mathrm{A}_{j}\right)_{j}$, l'effet des périodes (hiérachisé dans le carré latin, $j=3) ; C_{k}$, l'effet des traitements $(k=3) ; D_{k}$, l'effet des génisses $(l=6)$; et $\mathrm{e}_{i j k k}$, est l'erreur résiduelle du modèle. Les interactions carrés latins $x$ traitements et carrés latins $x$ génisses n'ont pas été significatives et n'ont pas été retenues dans le modèle final d'analyse.

Les données de cinétique horaire du comportement alimentaire ont été analysées selon un modèle hiérarchique, prenant en compte un effet temps et ses interactions avec les traitements, les génisses et les périodes. Les variables du comportement alimentaire mesurées pendant la nuit n'ont pas fait l'objet d'analyse de variance. Pour les données sur le fourrage proposé aux génisses, le modèle d'analyse a été comparable mais l'effet génisse n'a pas été pris en compte.

\section{RÉSULTATS}

\section{Conditions de déroulement de l'essai}

La température ambiante a été de $26^{\circ} \mathrm{C}$ en moyenne et a peu varié entre les périodes de mesure. La pluviométrie totale au cours de l'essai $(645 \mathrm{~mm})$ a été élevée pour la saison, par rapport à la moyenne des 30 dernières années $(280 \mathrm{~mm})$, mais cela est essentiellement dû à une valeur particulièrement élevée ( $367 \mathrm{~mm}$ ) au cours de la quatrième période.

La biomasse, la composition morphologique et la composition chimique du Dichanthium $\mathrm{sp}$ proposé aux génisses ont été comparables pour tous les traitements $(p>0,10$, tableau I). La biomasse a été en moyenne de 2,3 $\mathrm{t}$ de $\mathrm{MO} /$ ha et a été constituée de plus de $50 \%$ de feuilles vertes. La teneur en MS de l'herbe proposée a été assez élevée $(24,8 \%)$. Les teneurs en MAT et en NDF ont été de 11,6 et $75,9 \%$ MO respectivement et la digestibilité in vitro a été de 0,57. La surface et les quantités de Dichanthium $\mathrm{sp}$ proposées aux génisses n'ont pas été différentes entre les traitements (tableau I). La quantité de matière organique offerte a été de $11,6 \mathrm{~kg} / \mathrm{j}$ MO en moyenne ce qui est proche de la valeur souhaitée de $12 \mathrm{~kg}$.

\section{Quantités ingérées}

La récolte manuelle au pâturage a permis d'estimer l'excrétion fécale journalière avec un coefficient de variation résiduel de $8,4 \%$ (tableau II). La quantité de MO excrétée a été plus élevée de $20 \%$ environ $(p<0,001)$ avec le traitement S24 par rapport aux deux autres traitements (tableau II). La quantité de MO excrétée a varié de 0,99 à $1,15 \mathrm{~kg} / \mathrm{jour}$ 
Tableau I. Biomasse, composition morphologique et chimique du Dichantium proposé à six génisses créoles au pâturage.

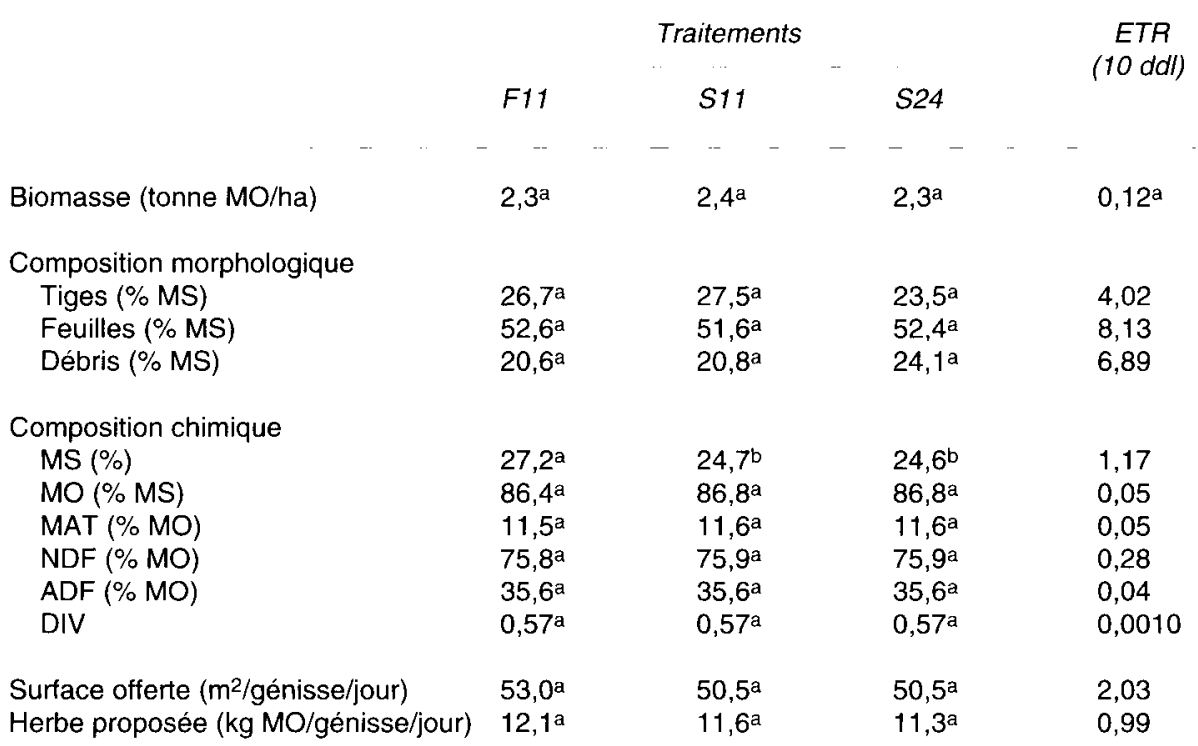

F11: Surface fractionnée disponible en 11 heures ; $S 11$ : surface entière disponible en 11 heures ; S24 : surface entière disponible en 24 heures; ETR : écart type résiduel de l'analyse de variance ; $a, b$ les valeurs suivies d'une même lettre ne sont pas significativement différentes au seuil de $p<0,05$.

selon les génisses $(p<0,01)$. La composition chimique des fèces, et en particulier la teneur en MAT, n'a pas varié entre les traitements $(p>0,10)$. Aussi, la digestibilité estimée du fourrage ingéré a été comparable pour tous les traitements et égale à 0,72 en moyenne.

Les quantités journalières de $\mathrm{MO}$ ingérée ont été plus élevées $(+0,6 \mathrm{~kg} / \mathrm{jour}, p<0,01)$ avec le traitement S24 et elles n'ont pas été significativement différentes entre les traitements $\mathrm{S} 11$ et $\mathrm{F} 11(p>0,10)$. Ces différences ont été maintenues lorsque les données ont été rapportées au poids métabolique des animaux. Les quantités ingérées ont varié entre les génisses de 3,6 à $4,4 \mathrm{~kg} /$ jour $(p<0,01)$. L'apport d'énergie nette a été plus élevé d'environ 0,5 UFL/jour pour le traitement $S 24(p<0,01)$. De plus, le poids vif des génisses a été légèrement plus élevé pour le traitement S24 (+ $3 \mathrm{~kg}$, $p<0,01$ ) et il n'a pas été différent entre les deux autres traitements.

\section{Comportement alimentaire}

Le temps de pâturage total et la répartition de l'activité d'ingestion pendant la journée (tableau III, fig 1) n'ont pas varié selon les traitements $(p>0,10)$. L'ingestion a représenté la moitié du nombre total d'observations, les génisses ayant pâturé en moyenne pendant 320 minutes. Le matin, l'activité d'ingestion a été intense dans l'heure qui a suivi l'arrivée des génisses 
Tableau II. Quantités et composition chimique des fèces récoltées sur les surfaces de pâturage, digestibilités et quantités ingérées de matière organique, apports en énergie (UFL) et poids vif de 6 génisses créoles au pâturage sur une prairie à Dichanthium.

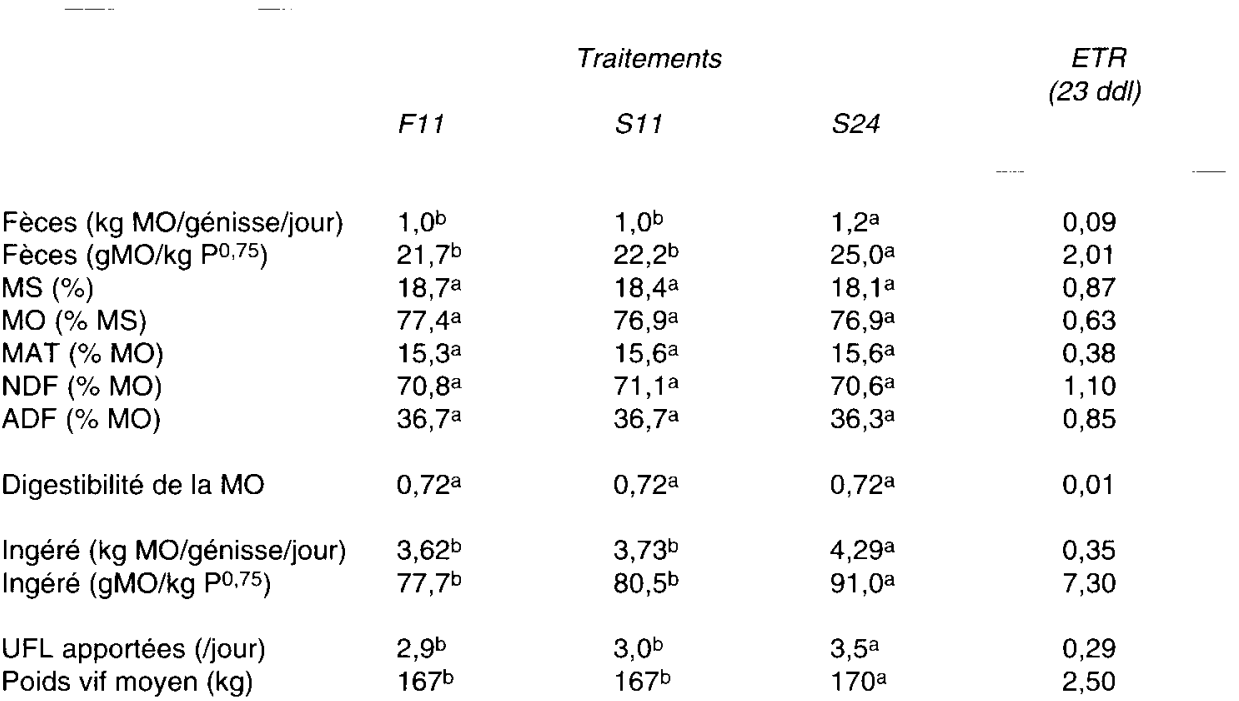

F11 : Surface fractionnée disponible en 11 heures. S11 : surface entière disponible en 11 heures : S24 : surface entière disponible en 24 heures ; ETR : écart type résiduel de l'analyse de variance ; $a, b$ les valeurs d'une même lettre ne sont pas significativement différent au seuil de $p<0,05$.

sur une nouvelle parcelle (55 minutes de pâturage entre $7 \mathrm{~h} 00$ et $8 \mathrm{~h} \mathrm{00)}$ puis elle a diminué régulièrement durant le reste de la matinée pour atteindre 20 minutes de pâturage entre $11 \mathrm{~h} 00$ et $12 \mathrm{~h} 00$ (fig 1). L'après midi il y a eu une reprise de l'activité d'ingestion entre $13 \mathrm{~h} 00$ et $14 \mathrm{~h} 00$ pour tous les traitements. Celle-ci a été légèrement plus marquée (+ 14 minutes) pour le traitement $F 11$, mais cette différence n'a pas été significative $(p>0,10)$. L'activité de pâturage a également été plus intense entre 16 h 00 et 17 h 00 . Dans le traitement $\$ 24$, les génisses ont pâturé pendant 100 minutes entre $18 \mathrm{~h} 00$ et $7 \mathrm{~h} 00$. Le pâturage nocturne proprement dit, entre $18 \mathrm{~h} 00$ et le lever du jour à $5 \mathrm{~h} 00$, a duré environ 60 minutes et a eu lieu essentiellement en milieu de nuit (entre $21 \mathrm{~h} 00$ et 2 h 00 , fig 2). L'activité de pâturage a redé- marré de manière assez intense au lever du soleil.

La fréquence de bouchées et leur nombre total pendant la journée n'ont pas varié selon les traitements (tableau III, $p>0,10$ ). En moyenne, la fréquence des bouchées a été de $53 /$ minute et les génisses ont réalisé 17000 bouchées entre $7 \mathrm{~h} 00$ et $18 \mathrm{~h} 00$. Le nombre de bouchées a varié selon les individus de 15000 à 18600 $(p<0,01)$. Dans le traitement S24, la fréquence des bouchées a été plus faible entre $18 \mathrm{~h} 00$ et $7 \mathrm{~h} 00$ que pendant le jour $(42 / \mathrm{min}, p<0,001)$ mais les génisses ont ainsi réalisé environ 4200 bouchées supplémentaires comparativement aux traitements $\mathrm{S} 11$ et $\mathrm{F} 11$. En revanche, la taille des bouchées a été similaire pour les trois traitements $(p>0,10)$ et égale à $230 \mathrm{mg}$ MO en moyenne. 
Tableau III. Nombre d'observations (nb obs) des activités de pâturage, rumination et autres activités, fréquence de bouchées, pendant le jour et la nuit de génisses créoles au pâturage sur une prairie à Dichanthium sp.

\begin{tabular}{|c|c|c|c|}
\hline \multicolumn{3}{|c|}{ Traitements } & \multirow{2}{*}{$\begin{array}{c}E T R \\
(23 d d l)\end{array}$} \\
\hline$F 11$ & S11 & S24 & \\
\hline $33^{a}$ & $33^{a}$ & $31^{a}$ & 2,5 \\
\hline $15^{\mathrm{a}}$ & $15^{\mathrm{a}}$ & $16^{a}$ & 2,1 \\
\hline $14^{a}$ & $14^{\mathrm{a}}$ & $15^{\mathrm{a}}$ & 3,1 \\
\hline $51,8^{\mathrm{a}}$ & $53,8^{a}$ & $53,7^{\mathrm{a}}$ & 2,22 \\
\hline 0 & 0 & 10 & $4,5\left(^{*}\right)$ \\
\hline & & 27 & $1,1\left(^{*}\right)$ \\
\hline & & 42 & $3,6\left({ }^{*}\right)$ \\
\hline & & 41,8 & $4,70\left(^{*}\right)$ \\
\hline
\end{tabular}

Valeurs moyennes de $7 \mathrm{~h} 00$ à $18 \mathrm{~h} 00$

Pâturage (nb obs)

Rumination (nb obs)

Autres activités ( $\mathrm{nb}$ obs)

Fréquences de bouchées ( $\mathrm{nb} /$ minute)

$15^{\mathrm{a}}$

Valeurs moyennes de $18 \mathrm{~h} 00$ à $7 \mathrm{~h} 00$

Pâturage (nb obs)

Rumination (nb obs)

Autres activités (nb obs)

Fréquences de bouchées (nb/minute)

F11 : Surface fractionnée, disponible en 11 heures; S11 : surface entière, disponible en 11 heures ; S24: surface entière disponible en 24 heures ; ETR : écart type résiduel de l'analyse de variance ; ${ }^{\star}$ ) écart type de la moyenne, aucune analyse de la variance n'a été effectuée; ${ }^{a, b}$ les valeurs d'une même ligne, suivies d'une même lettre ne sont pas significativement différentes au seuil de $p<0,05$.

minutes

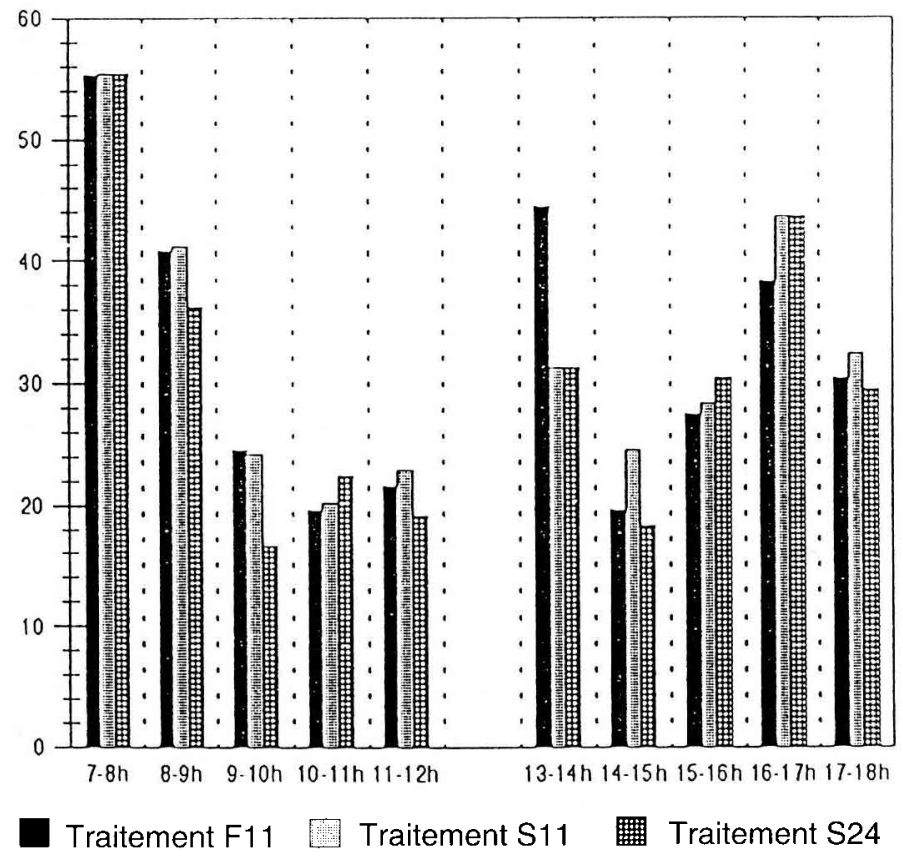

Fig 1. Durées horaires diurnes de pâturage de génisses créoles au pâturage. F11 : surface fractionnée, disponible en 11 heures. S11 : surface entière, disponible en 11 heures. S24 : surface entière disponible en 24 heures. Les barres d'une même tranche horaire (entre traitements) ne sont pas significativement différentes $(p>0,05)$. 
Fig 2. Durées horaires nocturnes de pâturage de génisses créoles au pâturage. S24 : surface entière disponible en 24 heures.

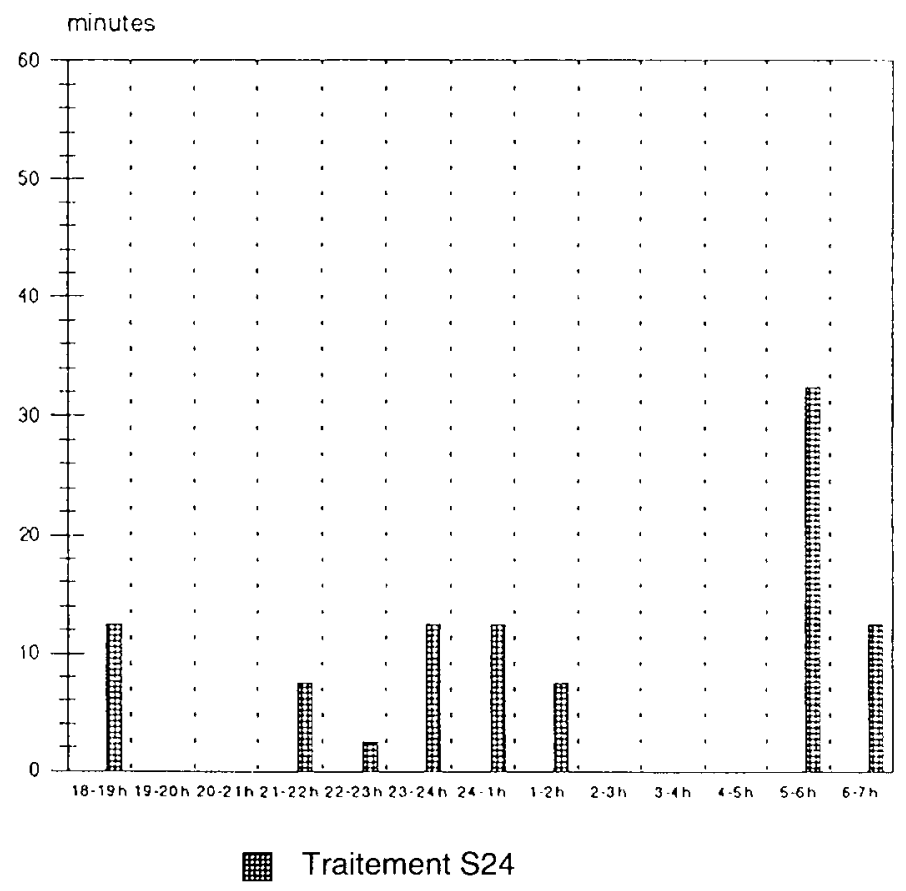

La durée de rumination pendant la phase diurne n'a pas été affectée par le traitement $(p>0,10)$. Elle a duré 150 minutes en moyenne. Lorsqu'elle a été enregistrée (traitement S24), la rumination nocturne a été de 260 minutes en moyenne.

\section{DISCUSSION}

\section{Caractéristiques du Dichanthium sp proposé et quantités ingérées}

La production de Dichanthium sp (160 kg MS/ha/jour) observée au cours de cet essai est plus importante que celle mesurée par Cruz et Schemoul (1991, 90 kg MS/ha/jour) en Guadeloupe avec des niveaux de fertilisation et des âges de repousse plus importants que dans notre étude. Les bonnes conditions hydriques combinées à la ferti- lisation azotée pourraient expliquer cette forte vitesse de croissance de la végétation dans nos conditions. La biomasse produite en 14 jours est en outre équivalente à celle du Digitaria decumbens, graminée plantée dans la zone et réputée comme étant très productive (Salette, 1970 ; Cruz et al, 1989). L'exploitation précoce des parcelles à 14 jours de repousse a permis d'obtenir un fourrage de bonne qualité pour la zone en période sèche. La biomasse disponible a été très feuillue avec un rapport feuille/tige de 2 et la teneur en MAT $(11,6 \% \mathrm{MO})$ a été comparable à celle mesurée par Doreau et Vivier (1979) et Xande (1985) en début de saison des pluies en Guadeloupe. La digestibilité du Dichanthium $\mathrm{sp}$ ingéré $(0,72)$ a été élevée et bien supérieure aux valeurs obtenues par Boval et al (1996) sur des repousses de 21 jours $(0,68)$. Elle a été comparable aux digestibilités les plus élevées mesurées en zone tropicale (Romero et Siebert, 1980). 
L'apport énergétique a été estimé à 3,3 UFL en moyenne, ce qui correspond assez bien aux besoins des animaux ( 3,1 UFLjour) compte tenu de la croissance pondérale moyenne mesurée au cours de l'essai $(0,43 \mathrm{~kg} / \mathrm{jour})$. La récolte manuelle des fèces et la détermination de la digestibilité à partir de la teneur en MAT des fèces semblent donc permettre des estimations cohérentes des apports nutritifs sur pâtures de Dichanthium sp. Par ailleurs, l'absence d'interactions entre les carrés latins et les génisses d'une part et les traitements d'autre part montre que la méthode a permis un classement répétable des effets. La méthode apparaît aussi relativement précise. La variabilité résiduelle de l'estimation des quantités de fèces a été de $8,4 \%$, ce qui est comparable aux valeurs généralement rapportées lorsque le flux de fèces est estimé par l'emploi de marqueurs externes (Le Du et Penning, 1982 ; Mélix et al, 1987).

\section{Durée et répartition du pâturage au cours du nycthémère}

Les durées de pâturage pendant la période diurne sont plus faibles que celles mesurées pour de jeunes bovins en Guadeloupe (400 minutes; Salas et al, 1990). La durée de pâturage entre 12 h 00 et 13 h 00 n'a pas été estimée, mais on peut supposer qu'elle était limitée en raison de l'abreuvement, de la récolte des fèces et des déplacements des génisses du traitement F11 effectués dans cet intervalle de temps.

Lorsque les génisses restent 24 heures au pâturage, il y a une reprise de l'activité au lever du jour, ce qui est conforme aux données d'Arnold (1981) et Hodgson (1982). L'activité de pâturage est réduite durant les heures les plus chaudes de la journée, elle est en revanche légèrement plus intense après $16 \mathrm{~h} 00$ avec la réduction de l'insolation et la baisse des tempéra- tures ainsi que Salas et al (1990) et Coulon (1984) l'avaient montré. Ce rythme circadien de l'activité a été ponctué par les interventions auprès des génisses. Le déplacement des génisses à $7 \mathrm{~h} 00$ sur une nouvelle parcelle a relancé l'activité de pâturage qui a été intense entre $7 \mathrm{~h} 00$ et $9 \mathrm{~h} 00$ quel que soit le traitement. Doreau (1979) a également mesuré une augmentation de la durée quotidienne de pâturage de $14,5 \%$, le jour du déplacement de vaches conduites à l'attache. De même, malgré l'insolation intense qui affecte l'ingestion des taurins (Barbier, 1987), il y a eu une activité de pâturage assez soutenue entre $13 \mathrm{~h} 00$ et $14 \mathrm{~h} 00$, vraisemblablement due à l'abreuvement (Jarrige, 1988 ; Fujihara et al, 1989).

\section{Effet sur l'ingestion de la durée de présence des animaux sur la savane}

Les quantités ingérées ont été plus élevées pour les génisses au pâturage toute la journée (traitement S24) par rapport à celles rentrées au piquet dortoir (traitements $S 11$ et F11). À l'auge, la limitation de la durée des repas se traduit également par une diminution des quantités ingérées (Chenost et Demarquilly, 1982). Le poids vif plus élevé des génisses avec le traitement $\$ 24$ est cohérent avec l'accroissement de l'ingestion. II traduit une augmentation du poids des contenus digestifs mais peut être aussi une croissance journalière plus élevée des animaux. Martin et Ruiz (1986) à Cuba ont en effet mis en évidence une réduction sensible du gain de poids (de 880 à 560 g/jour) lorsque les animaux sont parqués la nuit hors du pâturage. La durée limitée des périodes expérimentales ne permet cependant pas de conclure sur l'effet des traitements sur le gain de poids vif dans notre essai.

Cet accroissement de l'ingestion avec le traitement S24 est essentiellement lié au 
pâturage nocturne et dès le lever du soleil, qui, bien que moins important que pendant la journée, a permis aux animaux d'accroître le nombre de bouchées de 4200 (soit environ $25 \%$ ). En effet, les durées de pâturage et les fréquences de bouchées ont été comparables pendant la journée pour toutes les génisses et il est probable que les quantités ingérées entre $7 \mathrm{~h} 00$ et $18 \mathrm{~h} 00$ ont été similaires pour les trois traitements.

En revanche, les génisses placées au piquet dortoir n'ont pas accru leur durée de pâturage ou leur vitesse d'ingestion pendant la période diurne pour compenser le jeûne nocturne. En particulier la durée de pâturage le matin, dans les heures qui ont suivi l'arrivée sur une nouvelle parcelle, n'a pas été différente entre les traitements. En fait, l'effet du jeûne ne semble marqué que pour des jeûnes prolongés. Dougherty et al (1987) et Arias et al (1990) avaient observé un accroissement transitoire de la fréquence et la taille des bouchées de génisses au pâturage à l'attache après un jeûne de 16 heures, mais l'effet s'estompait après quelques heures de présence des animaux sur les parcelles. Seuls Greenwood et Demment (1988) ont mis en évidence une augmentation nette de la fréquence de bouchées et de l'ingestion après un jeûne très long de 36 heures.

\section{Effet du fractionnement de la surface à pâturer sur l'ingestion}

Le fractionnement de la surface offerte (traitement F11) n'a pas eu d'effet sur les quantités d'herbe ingérées pour une même durée de présence des animaux au pâturage. Ce résultat diffère de ceux généralement rapportés avec des animaux à l'auge, où le fractionnement des apports stimule l'ingestion en conditions tempérées (Chenost et Demarquilly, 1982) et tropicales (Ikhatua et al, 1987). It est cependant en bon accord avec les observations du comportement ali- mentaire. En moyenne, le temps de pâturage et la fréquence des bouchées des génisses disposant de deux petites surfaces ont été comparables à celles des autres génisses qui ont disposé d'une seule surface à pâturer.

En particulier, l'offre d'une nouvelle surface en début d'après midi, à 13 h 00 , n'a pas permis une relance importante de l'activité de pâturage. La durée de pâturage dans l'heure qui a suivi le déplacement n'a été plus élevée que d'une dizaine de minutes dans le traitement $\mathrm{F} 11$ comparé à $\mathrm{S} 11$. L'abreuvement réalisé entre $12 \mathrm{~h} 00$ et $13 \mathrm{~h} 00$ a pu masquer l'effet du déplacement sur l'ingestion, en relançant partiellement celle-ci pour tous les traitements (Jarrige, 1988). En effet l'activité d'ingestion a globalement augmenté entre $13 \mathrm{~h} 00$ et 14 h 00 pour tous les traitements. Par ailleurs, afin d'offrir les mêmes quantités d'herbe pour les trois traitements, les deux demi-surfaces offertes aux génisses du traitement F11 ont été très faibles $\left(24 \mathrm{~m}^{2}\right)$, ce qui a pu perturber le comportement des animaux. En particulier, le piétinement plus important a pu limiter l'appétibilité du fourrage (Brown et Evans, 1973). Dougherty et al (1992) avaient déjà mis en évidence une diminution de l'ingestion avec la taille des parcelles (50 versus $17 \mathrm{~m}^{2} /$ jour) mais, dans leur essai, cet effet était partiellement confondu avec les quantités offertes.

\section{CONCLUSION}

Le pâturage en 24 heures apparaît comme la conduite à l'attache la plus satisfaisante parmi celles que nous avons testées, pour accroître l'ingestion et sans doute les performances zootechniques. Cette conduite allège par ailleurs le temps de travail nécessaire aux déplacements quotidiens des animaux. II conviendrait de tester différentes modalités d'abreuvement au cours d'essais complémentaires. 


\section{REMERCIEMENTS}

Nous remercions toute l'équipe du domaine Inra de Gardel et plus particulièrement tous ceux qui ont participé à la réalisation des mesures et à l'observation des génisses au pâturage. Nous remercions également toute l'équipe du laboratoire Inra de Duclos, pour les analyses réalisées dans le cadre de ce travail. Enfin nous remercions $H$ Archimède et $G$ Aumont pour leur conseils lors de la rédaction de cet article.

\section{RÉFÉRENCES}

Arias JE, Dougherty CT, Bradley NW, Cornelius PL, Lauriault LM (1990) Structure of tall fescue swards and intake of grazing cattle. Agron J 82, 545548

Arnold GW (1981) Grazing behaviour. In : World Animal Science, B1. I. Grazing animals (FHW Morley, ed), Amsterdam, 79-104

Aumont G, Caudron I, Xande A (1991) Tables des valeurs alimentaires de fourrages tropicaux de la zone Caraïbe et de la Réunion (Inra-SRZ, ed), Guadeloupe, $199 \mathrm{p}$

Barbier MF (1987) Comportement alimentaire des zébus au pâturage en Guyane. Elev Méd Vét Pays trop $40,167-172$

Berbigier P (1988) Bioclimatologie des ruminants domestiques en zone tropicale. Inra Éd, Paris, $135 p$

Boval M, Borel H, Alexandre G, Aumont G, Xande A (1995) Exploitation traditionnelle de la savane naturelle par le bovin créole en Guadeloupe "la conduite à l'attache". Rev Elev Méd Vét Pays Trop (sous presse)

Boval M, Peyraud JL, Xande A, Aumont G, Copry O, Saminadin $G$ (1996) Évaluation d'indicateurs fécaux pour la prédiction de la digestibilité et des quantités ingérées de Dichanthium $\mathrm{sp}$ par des bovins créoles en Guadeloupe. Ann Zootech 45, 121-134

Brown KR, Evans PS (1973) Animal treading. A review of the late DB Edmond. New Zealand J Exp Agric 1, 217-226

Buisson C, Salas M (1985) Étude des systèmes d'élevage bovin traditionnel en Guadeloupe : aspects méthodologiques, premiers résultats, perspectives. In: Systèmes de production agricoles caribéens et alternatives de développement, colloque UAG-DAC, 9-11 mai 1985, 303-313

Chenost M, Demarquilly C (1982) Measurement of herbage intake by housed animals. In : Herbage Intake Handbook (JD Leaver, ed), British Grassland Society, 95-112
Coulon JB (1984) Comportement alimentaire de bovins croisés Charolais en milieu tropical humide. Rev Elev Méd vét Pays trop 37, 185-190

Cruz P. Alexandre G, Baudot H (1989) Cinétique de la croissance foliaire et stolonifère d'un peuplement de Digitaria decumbens au cours de la repousse. In : Proceedings of the XVIth International Grassland Congress, Nice, France, 499-500

Cruz P, Schemoul E (1991) Effet de l'azote sur l'expression du potentiel de croissance d'une prairie naturelle à base de Dichanthium aristatum en Guadeloupe (Antilles françaises). In : Proceedings of the IVth International Rangeland Congress, Montpellier, France, 360-363

Doreau M (1979) Comportement alimentaire au pâturage du bovin créole en Guadeloupe. Rev Elev Méd vét Pays Trop 32, 85-92

Doreau M, Vivier M (1979) La prairie naturelle à Dichanthium caricosum en Guadeloupe. I. Effet de la saison sur la production, la composition chimique et digestibilité in vitro. Agro Trop 23, 356-361

Dougherty CT, Bradley NW, Cornelius PL, Lauriault LM (1987) Herbage intake rates of beef cattle grazing Alfalfa. Agron J79, 1003-1008

Dougherty CT, Bradley NW, Lauriault LM, Arias JE, Cornelius PL (1992) Allowance intake relations of cattle grazing vegetative tall fescue. Grass Forage Sci 47 , 211-219

Fujihara T, Sumida M, Harumoto T (1989) The effect of water consumption on the eating and rumination behaviour of sheep fed only fresh or dried forage. Jap J Zootech Sci 30, 203-206

Greenwood GB, Demment MW (1988) The effect of fasting on short term cattle grazing behaviour. Grass Forage Sci 43, 377-386

Hodgson J (1982) Ingestive behaviour. In : Herbage Intake Handbook (JD Leaver, ed), British Grassland Society, 113-138

Ikhatua UJ, Ehoche OW, Umoh JE (1987) The influence of feeding frequency on feed intake, nutrient utilization and nitrogen metabolism in growing zebu cattle. J Agric Sci Camb 108, 639-642

Jamieson WS, Hodgson J (1979) The effect of daily herbage allowance and sward characteristics upon the ingestive behaviour and herbage intake of calves under strip-grazing management. Grass Forage Sci $34,261-271$

Jarrige $\mathrm{R}(1988)$ Ingestion et digestion des aliments. In: Alimentation des bovins, ovins et caprins, Inra Éd, 55-56

Le Du YLP, Penning PD (1982) Animal based techniques for estimating herbage intake. In: Herbage Intake Handbook (JD Leaver, ed), British Grasslands Society, 37-75

Martin PC, Ruiz R (1986) Weight gain and consumption of Holstein bulls grazing pangola grass (Digitaria decumbens Stent). Cuban J Agric Sci 20, 239-242 
Mélix C, Peyraud JL, Vérité R (1987) Utilisation de l'oxyde de chrome chez les vaches laitieres pour la prévision des quantités de fèces émises. I. Étude des variations du taux de récupération et ses conséquences sur l'estimation de la digestibilité et des quantités ingérées de rations d'herbe et d'ensilage de maïs. Reprod Nutr Dev 27, 215-216.

Romero A, Siebert BD (1980) Seasonal variations of nitrogen and digestible energy intake of cattle on tropical pasture. Aust J Agric Res 31, 393-400

SAS (1988) SAS user's guide. Statistics. SAS Inst Inc, Cary, NC, États-Unis

Salas M, Biessy G, Magne E (1990) Effet du mode de conduite au pâturage et de la complémentation sur le comportement alimentaire des bovins en Guadeloupe. Rev Elev Méd vét Pays trop 43, 381-386

Salas M, Sheikboudou C (1988) Alimentation des bovins en saison sèche dans les systèmes d'élevage guadeloupéen : analyse des pratiques paysannes. Cahier Rech Dév 17, 54-63
Salette JE (1970) Les cultures fourragères tropicales et leurs possibilités d'intensification. Fourrages 43, 91107

Tilley JMA, Terry RA (1963) A two stage technique for in vitro digestion of forage crops. J Br Grassid Soc 18 , 104-111

Troccon JL (1987) Recommandations alimentaires pour les veaux et les génisses d'élevage. Bull Tech CRVZ Theix INRA 70, 167-172

Van Soest PJ (1963) Use of detergents in the analysis of fibrous feeds. 2. A rapid method for the determination of fibre and lignin. J Assoc Off Agric Chem 46, 829835

Xande A (1985) La productivité des pâturages : problèmes posés et intérêt de quelques techniques pour améliorer la productivité dans le cadre des petites exploitations. In : Systèmes de production agricoles caribéens et alternatives de développement, colloque UAG-DAC, 9-11 mai 1985, 369387 